\title{
Recent Advances in Methyltransferase Biocatalysis
}

DOI:

10.1016/j.cbpa.2017.01.020

\section{Document Version}

Accepted author manuscript

Link to publication record in Manchester Research Explorer

\section{Citation for published version (APA):}

Bennett, M., Shepherd, S., Cronin, V., \& Micklefield, J. (2017). Recent Advances in Methyltransferase Biocatalysis. Current Opinion in Chemical Biology, 37, 97-106. https://doi.org/10.1016/j.cbpa.2017.01.020

\section{Published in:}

Current Opinion in Chemical Biology

\section{Citing this paper}

Please note that where the full-text provided on Manchester Research Explorer is the Author Accepted Manuscript or Proof version this may differ from the final Published version. If citing, it is advised that you check and use the publisher's definitive version.

\section{General rights}

Copyright and moral rights for the publications made accessible in the Research Explorer are retained by the authors and/or other copyright owners and it is a condition of accessing publications that users recognise and abide by the legal requirements associated with these rights.

\section{Takedown policy}

If you believe that this document breaches copyright please refer to the University of Manchester's Takedown Procedures [http://man.ac.uk/04Y6Bo] or contact uml.scholarlycommunications@manchester.ac.uk providing relevant details, so we can investigate your claim.

\section{OPEN ACCESS}




\section{Recent Advances in Methyltransferase Biocatalysis}

Matthew R. Bennett, Sarah A. Shepherd, Victoria A. Cronin, Jason Micklefield*

School of Chemistry \& Manchester Institute of Biotechnology

University of Manchester

131 Princess Street

Manchester

United Kingdom

M1 7DN

*corresponding author: jason.micklefield@manchester.ac.uk

Abstract. S-adenosyl methionine dependent methyltransferases are ubiquitous in nature, methylating a vast range of small molecule metabolites, as well as biopolymers. This review covers the recent advances in the development of methyltransferase enzymes for synthetic applications, focusing on the methyltransferase catalysed transformations with $S$-adenosyl methionine analogs, as well as non-native substrates. We discuss how researchers have used metabolic engineering approaches to enhance $S$-adenosyl methionine production in vivo. Enzymatic approaches that enable the more efficient generation of $S$-adenosyl methionine analogs, including more stable analogs, will also be described; this has expanded the biocatalytic repertoire of methyltransferases from methylation to a broader range of alkylation reactions. The review also examines how the selectivity of the methyltransferase enzymes can be improved through structure guided mutagenesis approaches. Finally we will discuss how methyltransferases can be deployed in multi-enzyme cascade reactions and suggest future challenges and avenues for further investigation.

Introduction. S-adenosylmethionine (AdoMet) dependent methyltransferases (MTases) are ubiquitous in nature, methylating biopolymers including nucleic acids and proteins, as well as small molecule metabolites. MTases typically catalyse the transfer of a methyl group from AdoMet to C-, $\mathrm{O}-, \mathrm{N}-$, or $\mathrm{S}$ - centred nucleophiles of an acceptor substrate. These $\mathrm{S}_{\mathrm{N}} 2$ reactions normally proceed with high regioselectivity and in some cases generate new stereogenic centres with complete stereoselectivity. The methyl groups introduced by MTases are often also important for the function or bioactivity of the resulting methylated product. For example, MTases are particularly common in secondary metabolism, methylating a wide range of biosynthetic scaffolds, which can affect the physicochemical and biological properties of the resulting natural products. [1-3] In recent years, 
there has been interest in exploiting the selectivity and benign operating conditions of MTases for synthetic applications, including the derivatization of non-native substrates. For example, in synthetic chemistry, methyl substituents are often introduced to optimize lipophilicity, binding affinity and other properties in the development of pharmaceuticals. [4] However, the commonly used synthetic (non-enzymatic) alkylating agents are toxic and do not usually afford good regio-, chemo- or stereo-selectivity, which makes cleaner and more selective biocatalytic methylation approaches particularly attractive.

Many MTases have also been shown to accept AdoMet analogs (e.g. 1b-m, Figure 1A) with alternative, $S$-alkyl substituents, facilitating the transfer of a range of functional groups to acceptor substrates. [2,3,5-10] Moreover, the transfer of an orthogonally reactive functional group from an AdoMet analog (e.g. 1j-m, Figure $1 \mathrm{~A}$ ) to proteins or nucleic acid substrates has found application as a biomolecular labelling tool. [7,11-14] Nevertheless, the production and recycling of AdoMet (1a) and AdoMet analogs remains a challenge. The development of MTases as biocatalysts for transformations of non-native substrates is also an area of interest. In this review, we will focus on recent research to generate AdoMet analogs, as well as the characterization and engineering of new MTase biocatalysts. $[3,15,16]$ This review will also describe new cascade reactions that include MTases. $[17,12]$ For earlier work in this field, the reader is referred to the previous review by Struck et al, which describes research on MTases prior to 2012. [18]

Co-factor generation. One of the main limitations of utilizing MTases in vitro is the availability of AdoMet (1a). To date, no effective in vitro AdoMet recycling system has been developed. In light of this, the deployment of MTases for larger scale methylation reactions is most viable in vivo. For example, a mammalian catechol $O$-methyl transferase (COMT) has been used to produce vanillin (a commercially important fine chemical) in engineered strains of yeast or E. coli. $[19,20]$ The deregulation of AdoMet biosynthesis in $E$. coli has been shown to increase levels of AdoMet and improve yields of vanillin.[19] The production of AdoMet was upregulated via the over expression of methionine biosynthetic genes and the deletion of the transcriptional repressor (met)), which normally regulates methionine production. [19] Metabolic engineering approaches could provide valuable engineered host strains, with elevated AdoMet production, for expression of other MTases.

A wide range of MTases can utilize AdoMet analogs with alternative substituents in place of the S-methyl group (e.g. 1b-m, Figure $1 \mathrm{~A}$ ). The early studies utilized AdoMet analogs that were prepared synthetically via alkylation of S-adenosyl-L-homocysteine (AdoHcy, 2) [14,21] However, the synthetic approach results in a mixture of sulfonium epimers, which require separation as only the (S)-configured diastereoisomer is normally accepted by MTases and the opposite $(R)$ - 
diastereoisomer, along with residual AdoHcy (2) can inhibit MTases. [3] More recently, enzymatic approaches have been developed to produce AdoMet analogs, which overcome some of the difficulties associated with the synthetic method (Figure 1A). For example, the halogenase enzymes SalL from Salinispora tropica [22] and 5'-fluoro-5'-deoxyadenosine synthase (FDAS) from Streptomyces cattleya [23], which cleave AdoMet to give L-methionine (L-Met) and 5'-chloro or 5'fluoro-5'deoxyadenosine (CIDA 3 or FDA 4) respectively, can be utilised in the reverse direction to produce AdoMet or AdoMet analogs from the corresponding L-Met (5a) or L-Met derivative (5b-m, Figure 1A). [15,24] Lipson et al exploited recombinant SalL, with CIDA and L-Met, to produce AdoMet in situ demonstrating methylation of DNA and the glycopeptide antibiotic teicoplanin using the Hhal and MtfA MTases, respectively. [24] In a more comprehensive study, Thomsen et al explored the activity of SalL and FDAS (wild type and active site mutants) with a series of L-Met analogs and CIDA or FDA as substrates. [15] SalL was determined to be active with L-Met and five L-Met analogs (5b-f) with activity generally decreasing with increasing size of the substituent. [15] These enzymes marked significant progress, although they did present a number of drawbacks due to poor kinetics and the need for expensive precursors (CIDA or FDA).

Methionine adenosyltransferases (MAT, also known as AdoMet synthetases) offer an alternative enzymatic approach to produce AdoMet analogs (Figure 1A). The MAT enzyme utilises LMet and adenosine triphosphate (ATP) for the biosynthesis of AdoMet, and given that ATP is cheaper, more accessible and can also be recycled it offers significant advantages over the use of CIDA or FDA with halogenases. [25] MAT enzymes from different organisms have been shown to accept a wide range of L-Met analogs (5b-e \& $\mathbf{5 g - j})$. $[3,13]$ Wang et al also used the the crystal structure of the human MAT2A (hMAT2A) to make a series of mutations to hMAT2A that increased the size of the L-Met binding pocket to accept larger L-Met analogs.[13,26] Of the 17 mutants examined the I117A hMAT2A mutant was found to have the best activity towards the bulky methionine analogs $S$-(E)-pent-2-en-4-ynyl homocysteine (5k), $S$-(E)-hex-2-en-5-ynyl homocysteine (5I) and S-4-propargyloxy-but-2-enyl homocysteine (5m). [13] Singh et al took a different approach by exploring the diversity nature provides. [3] Five MATs from bacterial, archaeal and mammalian origins were assessed with $44 \mathrm{~L}-\mathrm{Met}$ or L-selenomethionine analogs. hMAT2A was determined to be the most promiscuous, accepting 29 L-Met analogs, of which ten exhibited conversions over $50 \%$ to the corresponding AdoMet derivatives. The MAT from the thermophile Methanocaldococcus jannaschii was also shown to display good activity with L-Met analogs. In general, Se-adenosyl-Lselenomethionine analogs were more effective than the AdoMet analogs in subsequent MTase catalysed reactions. [3] 
The use of halogenases and MAT offers several advantages over synthetic methods for producing AdoMet analogs. The enzymatic approaches enable the efficient and selective production of the single active sulfonium (or selenonium) diastereomer in situ, with good yields, obviating the need for co-factor purification steps prior to MTase catalysed alkylation reactions. The ability to couple enzymatic co-factor generation with subsequent MTase biocatalysis is also particularly important given the instability of AdoMet (analogs) under typical enzymatic reaction conditions. [1] AdoMet spontaneously decomposes in buffers at physiological $\mathrm{pH}$ and ambient temperatures with a relatively short half-life $\left(t_{1 / 2}=17 \pm 0.4 \mathrm{~h}\right)$, whilst some AdoMet analogs are even more susceptible to rapid degradation. [27] The two main AdoMet degradation pathways are side chain cyclisation, which generates 5'-deoxy-5'-methylthioadenosine (MTA, 6) that can inhibit MTases, and depurination to give $S$-pentosylmethionine (7, Figure 1B). To overcome co-factor degradation, three AdoMet isosteres (8-10) with increased stability were prepared enzymatically using hMAT2A (Figure 1B) and all were shown to be functional as cofactors, in methylation reactions with a prototypical Class I MTase (DnrK). [28] Notably the 7dzAdotMet (10) isostere, prepared enzymatically from 7deazoadenosine and the tetrazole analog of L-Met, showed almost complete resistance to both intramolecular cyclization and depurination. Further work is now necessary to establish if derivatives of $7 \mathrm{dzAdotMet}(\mathbf{1 0})$, with alternative $S$-alkyl substituents, can facilitate a wider range of MTase catalysed alkylation reactions.

MTase structure, selectivity and coupled transformations. In addition to the progress that has been made on the generation of AdoMet and analogs, there has been recent progress on the characterisation and applications of MTases, including the development of cascades combining MTases with other enzymes. Noteably, X-ray crystal structures have been solved for a number of interesting $N$-Methyltransferases ( $N$-MTase), providing insights into substrate recognition that might be used to broaden the biocatalytic scope of these enzymes. For example Ccbj from Streptomyces caelestis, which methylates a proline residue of its substrate $N$-demethylcelesticetin (Figure 2A). [29] The methylated product celesticetin (11) is a lincosamide antibiotic with $N$-methylation proving vital for its activity. Recently, the crystal structure of Ccbj was solved by Bauer et al who identified three key residues in this $\mathrm{N}$-MTase, Y9 (catalytic base), Y17 and F117 (substrate binding residues), as well as a large substrate binding site (Figure 2B). [30] It is envisaged that the large and potentially promiscuous substrate binding site of Ccbj could enable future studies to generate a library of clinically important $N$-methyl lincosamide antibiotics. $[29,30]$

BamL from Bacillus amyloliquefaciens dimethylates an $N$-terminal amino group of thiazole/oxazole modified peptide precursor in the biosynthesis of plantazolicin (Figure 2A).[31] 
Again the activity of plantazolicin is drastically reduced without the $\mathrm{N}$-methylation. Structural studies identified BamL to have a catalytic base (Y182), and a narrow substrate channel, which accounts for its limited substrate range (Figure 2B). Initial studies investigated whether the plantazolicin mimic bradykinin could be a potential substrate, but this proved unsuccessful. However, with structural knowledge in hand, shorter synthetic tetrapeptide analogs of natural peptide (14mer) substrate were shown to be methylated by BamL and the resulting products (e.g. 12) displayed antimicrobial activity against methicillin-resistant Staphylococcus aureus (MRSA). $[31,32]$

The crystal structures of two MTase enzymes involved in benzylisoquinoline alkaloid biosynthesis have also recently been elucidated, the pavine- $N$-methyltransferase (PNMT) and norcoclaurine-6-O-methyltransferase (6OMT) (Figure 2B). [33,34] The characterization of MTases from these pathways could be useful in developing biocatalytic approaches for production of alkaloid derivatives, which have been widely used as pharmaceuticals. Robin et al solved the structure of 6-OMT which is known to methylate at the $6-\mathrm{OH}$ position of norcoclaurine producing coclaurine (14). $[34,35]$ Based on comparisons with the structurally similar $O$-methyltransferases $(O$ MTases) caffeic acid-O-methyltransferase (CaOMT) and isoflavone-O-methyltransferase (IOMT), the residues F119, W123, 1114, F140 and F162 of 6-OMT were determined to be important for substrate specificity. [34] Hence, mutagenesis of these key residues may lead to an expanded substrate range. Another structurally similar O-MTase, PAMTH1, from the plant Podospora anserine, methylates the isoflavone myricetin. $[36,37]$ This enzyme may provide access to a range of methylated flavonoids (15), which have been shown to have anti-inflammatory and anti-cancer properties. [38] The improved structural understanding of both $O$-MTase and $N$-MTase active sites can now be used to expand the substrate range of these enzymes, increasing their value as biocatalysts with non-native substrates (Figure 2B). [1,12,16,32,34,36]

The mammalian catechol $O$-methyltransferase (COMT) is involved in catabolism of catecholamines such as dopamine. The active site of COMT is close to the enzyme surface, enabling a variety of non-native catechol substrates to be methylated (16). The substrate promiscuity of COMT has been exploited for production of vanillin. [19,39,40] However, COMT catalysed methylation of vanillin precursor 3,4-dihydroxybenzaldehyde (19) results in a mixture of vanillin (20) and isovanillin (21) regioisomers (Figure $3 \mathrm{~A}$ ). Based on the structure of the COMT active site (Figure 3B), Law et al conducted mutagenesis studies leading to a mutant Y200L, which improved COMT regioselectivity from $58 \%$ to $90 \%$ regioisomeric excess in favour of the desired meta-methylated product $\mathbf{2 0}$ whilst maintaining near WT activity. The improved regioselectivity of the Y200L mutant was also exploited to effect a one-pot tandem enzymatic synthesis of the commercially important 
fine chemical ethyl vanillin in a 58\% isolated with ethyl-AdoMet (1b) generated in situ (Figure 1A). [16] Law et al also reported that the different oligomeric forms of COMT exhibit different regioselectivities, with the wild type COMT dimer exhibiting higher meta-selectivity than the monomeric form of the enzyme. [16] X-ray crystallography revealed that the COMT dimeric form is stabilised through domain swapping of the C-termini, which has also been observed in the structures of other O-MTases including CaOMT, IOMT and PAMTH1.[16,36,41,42]

The lack of regioselectivity observed with the wild type COMT, is unusual. Most MTases exhibit exquisite regioselectivity. For example in the biosynthesis of the immunosuppressant rapamycin three $O$-MTase enzymes RapM, RapQ and Rapl are predicted, based on gene deletion experiments, to catalyse the selective methylation of C-16, C-27 and C-39 hydroxyl groups respectively, of rapamycin precursors in Streptomyces rapamycinicus [43]. In recent studies, the recombinant RapM enzyme was shown to selectively methylate desmethyl rapamycin precursors at the 16-OH position (17, Figure 2A) [1]. Moreover RapM was shown to accept AdoMet derivatives, transferring alternative substituents to the $16-\mathrm{OH}$ position [1]. Similar approaches have also been used in the alkyl diversification of other bioactive natural product scaffolds, [2,3] providing strategies for generating natural product derivatives with altered physicochemical properties and bioactivity, or installing orthogonal handles to pull down in vivo biological targets (binding partners). [1-3]

The alkyl diversification approach [1-3] is dependent on nature having already evolved an MTase for the target molecule of interest. In light of this Struck et al. developed a more general approach, which enables the alkyl diversification of a wide range of substrates possessing a phenolic moiety (Figure 4A). [12] The approach utilizes the enzyme tyrosinase to selectively hydroxylate the phenolic moiety to a give a catechol, which can then be alkylated with COMT and an AdoMet analog. Both tyrosinase and COMT are relatively promiscuous and when used in tandem, can effect selective alkoxylation of tyrosine residues in a wide range of synthetic or natural peptides including peptide hormones, cyclic peptide antibiotics and the anticancer agent goserelin (22), (Figure 4B). The tyrosinase-COMT cascade was also shown to derivatize tyrosine residues in the $\mathrm{N}$ - or $\mathrm{C}$-terminal regions of proteins, providing a novel posttranslational modification system, which may be useful for functionalization of proteins for labeling, pull-down, drug-conjugation and other potential applications (Figure 4C).

Whilst $O$ - and $N$-MTases have been most widely studied, there are a number of $C$-MTases that have been explored as potential biocatalysts. [21] For example, C-MTases that catalyse the stereoselective $\beta$-methylation of $\alpha$-ketoacid substrates are of interest, and in combination with aminotransferase enzymes provide access to a range of non-proteinogenic $\beta$-methyl- $\alpha$-amino acids. $[17,44-46]$ Many synthetic peptidomimetics and other pharmaceuticals are derived from $\beta$-methyl- 
$\alpha$-amino acid building blocks, which are currently prepared using expensive multi-step synthetic processes. It is envisaged that the $\alpha$-ketoacid $C$-MTases could be used in combination with aminotransferases to provide a more efficient and cleaner route to $\beta$-methyl- $\alpha$-amino acids and derivatives. Recently Zou et al solved the X-ray crystal structure of an $\alpha$-ketoacid C-MTase,from the mannopeptimycin producer Streptomyces hygroscopicus NRRL 30439 [47]. This C-MTase, phenylpyruvate-C3-methyltransferase (MppJ), methylates phenylpyruvate to generate (3R)-3methylphenylpyruvic acid (18, Figure 2A) which can be converted to the $(2 S, 3 R)$-3-methylphenylalanine using an aminotransferase enzyme. The MppJ structure reveals a unique non-haem iron centre that binds to and activates the $\alpha$-ketoacid substrate for stereoselective methylation. These structural insights can help guide the engineering of the other non-haem iron dependent $\alpha$ ketoacid C-MTase, [47] which can enable the biocatalytic production of a wide range of $\beta$-methyl- $\alpha$ amino acid building blocks. Moreover, the MppJ structure also guided mutagenesis studies leading to MppJ variants with $O-M T a s e$ activity. [47]

Conclusions and Future Perspectives. Despite the substantial progress in AdoMet-dependent MTase biocatalysis, the high cost of AdoMet and feedback inhibition caused by cofactor by-products, such as AdoHyc (2) and MTA (6), still prove limiting. In order for the process to be economically and industrially viable a successful method to regenerate AdoMet is necessary. Enzymatic recycling systems have already been developed for several cofactors, including ATP in phosphoryl transfer reactions $[48,49]$ and both $\mathrm{NAD}(\mathrm{P})^{+}$and $\mathrm{NAD}(\mathrm{P}) \mathrm{H}$ in oxidoreductions. [48,50-52] However, no effective method to regenerate AdoMet in vitro has yet been reported, emphasising an important area for future work.

Both methionine adenosyltransferases MATs and halogenases (SalL and FDAS) have been explored for the synthesis of AdoMet analogs. [3,15,53,54]. An alternative way to enzymatically produce the AdoMet derivative, carboxy-S-adenosyl-L-methionine (carboxy-AdoMet) (23) recently emerged (Figure 5), through the unexpected finding that E.coli $\mathrm{CmoA}$ catalyses the conversion of AdoMet to carboxy-AdoMet $(\mathbf{2 3})$. $[9,10]$ Notably, carboxy-AdoMet provides the first example of a naturally occurring and physiologically relevant modified AdoMet analog.[9] This study also raises the possibility that a number of enzymes may be incorrectly annotated as AdoMet-dependent MTases but instead my utilize carboxy-AdoMet, which could subsequently be used in downstream MTase reactions. $[6,9]$

Expanding the range of chemical moieties that MTases can transfer would undoubtedly improve the versatility of this enzyme class. Several MTases accept AdoMet analogs with alternative functional groups in place of the $S$-methyl group;[18] however activity decreases considerably towards more bulky analogs. [14] This prompted MTase protein engineering studies focused on 
accommodating larger analogs with downstream applications for profiling protein methylation. $[7,8,11,13,53]$ Moreover, an MTase which is more selective for AdoMet analogs, over AdoMet, would be desirable for in vivo or whole cell biotransformations, where analogs must outcompete the native cofactor (the cellular concentration of AdoMet in E.coli is ca. $180 \mu \mathrm{M}$ ). [10,55]

As demonstrated in this review, AdoMet-dependent MTases show significant and expanding scope for biocatalytic applications, with most recent progress in enzymatic AdoMet analog synthesis, the development of valuable tandem reactions and the design of stable AdoMet isosteres. [28] Structural studies have proved key in developing this field, enabling the MTase substrate scope to be assessed and guiding mutagenesis to expand the substrate range, cofactor range and even regioselectivity of this enzyme class. $[13,16]$ Perceived future work would further expand the range of AdoMet analogs which can be generated by MATs and also utilised by MTases, whilst retaining activity comparable to the wild-type enzyme/native cofactor reactions. Identifying a range of MTases with useful substrate targets for API generation, would also add value to the field. An MTase-halogenase hybrid enzyme (AoiQ), for example, was recently reported to catalyse dichlorination of a diaporthin substrate followed by bis-O-methylation; such hybrid enzymes could prove valuable, considering the importance of both methylation and halogenation in modifying biological activity. [56] Finally, the development of an efficient AdoMet regeneration system would greatly increase the viability of MTase biocatalysis for larger scale and industrial applications.

\section{Acknowledgements}

The authors laboratory work is supported by BBRSC grants (BB/K00199X/1 \& BB/L002299/1).

\section{References}

1. Law BJC, Struck A, Bennett MR, Wilkinson B, Micklefield J: Site-specific bioalkylation of rapamycin by the RapM 16-0-methyltransferase [Internet]. Chem. Sci. 2015, 6:2885-2892.

2. Winter JM, Chiou G, Bothwell IR, Xu W, Garg NK, Luo M, Tang Y: Expanding the structural diversity of polyketides by exploring the cofactor tolerance of an inline methyltransferase domain. Org. Lett. 2013, 15:3774-3777.

3. Singh S, Zhang J, Huber TD, Sunkara M, Hurley K, Goff RD, Wang G, Zhang W, Liu C, Rohr J, et al.: Facile chemoenzymatic strategies for the synthesis and utilization of S-adenosyl-Lmethionine analogues. Angew. Chemie - Int. Ed. 2014, 53:3965-3969.

4. Schönherr $\mathrm{H}$, Cernak T: Profound methyl effects in drug discovery and a call for new C-H methylation reactions. Angew. Chemie - Int. Ed. 2013, 52:12256-12267.

5. Bothwell IR, Luo M: Large-Scale, Protection-Free Synthesis of Se -Adenosyl- I selenomethionine Analogues and Their Application as Cofactor Surrogates of Methyltransferases. Org. Lett. 2014, 16:3056-3059.

6. Byrne RT, Whelan F, Aller P, Bird LE, Dowle A, Lobley CMC, Reddivari Y, Nettleship JE, Owens RJ, Antson AA, et al.: S-Adenosyl-S-carboxymethyl-I-homocysteine: A novel cofactor found in the putative tRNA-modifying enzyme CmoA. Acta Crystallogr. Sect. D Biol. Crystallogr. 
2013, 69:1090-1098.

7. Islam K, Bothwell I, Chen Y, Sengelaub C, Wang R, Deng H, Luo M: Bioorthogonal Profiling of Protein Methylation Using Azido Derivative of S -Adenosyl- I-methionine. J. Am. Chem. Soc. 2012, 134:5909-5915.

8. Islam K, Chen Y, Wu H, Bothwell IR, Blum GJ, Zeng H, Dong A, Zheng W, Min J, Deng H, et al.: Defining efficient enzyme-cofactor pairs for bioorthogonal profiling of protein methylation. . Proc. Natl. Acad. Sci. U. S. A. 2013, 110:16778-83.

9. Kim J, Xiao H, Bonanno JB, Kalyanaraman C, Brown S, Tang X, Al-Obaidi NF, Patskovsky Y, Babbitt PC, Jacobson MP, et al.: Structure-guided discovery of the metabolite carboxy-SAM that modulates tRNA function. Nature 2013, 498:123-126.

10. Kim J, Xiao H, Koh J, Wang Y, Bonanno JB, Thomas K, Babbitt PC, Brown S, Lee YS, Almo SC: Determinants of the $\mathrm{CmoB}$ carboxymethyl transferase utilized for selective tRNA wobble modification. Nucleic Acids Res. 2015, 43:4602-4613.

11. Wang $R$, Zheng $W, Y u H$, Deng $H$, Luo $M$ : Labeling substrates of protein arginine methyltransferase with engineered enzymes and matched S-adenosyl-I-methionine analogues. J. Am. Chem. Soc. 2011, 133:7648-7651.

12. Struck AW, Bennett MR, Shepherd SA, Law BJC, Zhuo Y, Wong LS, Micklefield J: An Enzyme Cascade for Selective Modification of Tyrosine Residues in Structurally Diverse Peptides and Proteins. J. Am. Chem. Soc. 2016, 138:3038-3045.

13. Wang R, Islam K, Liu Y, Zheng W, Tang H, Lailler N, Blum G, Deng H, Luo M: Profiling genomewide chromatin methylation with engineered posttranslation apparatus within living cells. J. Am. Chem. Soc. 2013, 135:1048-1056.

14. Dalhoff C, Lukinavičius G, Klimaauskas S, Weinhold E: Direct transfer of extended groups from synthetic cofactors by DNA methyltransferases. Nat. Chem. Biol. 2006, 2:31-32.

15. Thomsen M, Vogensen SB, Buchardt J, Burkart MD, Clausen RP: Chemoenzymatic synthesis and in situ application of S-adenosyl-I-methionine analogs. Org. Biomol. Chem. 2013, 11:7606.

16. Law BJC, Bennett MR, Thompson ML, Levy C, Shepherd SA, Leys D, Micklefield J: Effects of Active-Site Modification and Quaternary Structure on the Regioselectivity of Catechol-OMethyltransferase. Angew. Chemie - Int. Ed. 2016, 55:2683-2687.

17. Huang Y-T, Lyu S-Y, Chuang P-H, Hsu N-S, Li Y-S, Chan H-C, Huang C-J, Liu Y-C, Wu C-J, Yang W$B$, et al.: In vitro Characterization of Enzymes Involved in the Synthesis of Nonproteinogenic Residue ( $2 \mathrm{~S}, 3 \mathrm{~S}$ )- $\beta$-Methylphenylalanine in Glycopeptide Antibiotic Mannopeptimycin. ChemBioChem 2009, 10:2480-2487.

18. Struck AW, Thompson ML, Wong LS, Micklefield J: S-Adenosyl-Methionine-Dependent Methyltransferases: Highly Versatile Enzymes in Biocatalysis, Biosynthesis and Other Biotechnological Applications. ChemBioChem 2012, 13:2642-2655.

19. Kunjapur AM, Hyun JC, Prather KLJ: Deregulation of $\mathbf{S}$ - adenosylmethionine biosynthesis and regeneration improves methylation in the $\mathrm{E}$. coli de novo vanillin biosynthesis pathway. Microb. Cell Fact. 2016, doi:10.1186/s12934-016-0459-x.

20. Hansen EH, Møller BL, Kock GR, Bünner CM, Kristensen C, Jensen OR, Okkels FT, Olsen CE, Motawia MS, Hansen J: De novo biosynthesis of Vanillin in fission yeast (schizosaccharomyces pombe) and baker's yeast (saccharomyces cerevisiae). Appl. Environ. Microbiol. 2009, 75:2765-2774.

21. Stecher H, Tengg M, Ueberbacher BJ, Remler P, Schwab H, Griengl H, Gruber-Khadjawi M: Biocatalytic Friedel-Crafts alkylation using non-natural cofactors. Angew. Chemie - Int. Ed. 2009, 48:9546-9548.

22. Eustáquio AS, Pojer F, Noel JP, Moore BS: Discovery and characterization of a marine bacterial SAM-dependent chlorinase. Nat. Chem. Biol. 2008, 4:69-74.

23. O'Hagan D, Schaffrath C, Cobb SL, Hamilton JTG, Murphy CD: Biochemistry: biosynthesis of an organofluorine molecule. Nature 2002, 416:279. 
24. Lipson JM, Thomsen M, Moore BS, Clausen RP, La Clair JJ, Burkart MD: A Tandem Chemoenzymatic Methylation by S-Adenosyl-L-methionine. ChemBioChem 2013, 14:950953.

25. Andexer JN, Richter M: Emerging enzymes for ATP regeneration in biocatalytic processes. ChemBioChem 2015, 16:380-386.

26. Komoto J, Yamada T, Takata Y, Markham GD, Takusagawa F: Crystal structure of the S adenosylmethionine synthetase ternary complex : a novel catalytic mechanism of $\mathbf{S}$ adenosylmethionine synthesis from ATP and Met. Biochemistry 2004, 43:1821-1831.

27. Lukinavičius G, Tomkuvienè M, Masevičius V, Klimašauskas S: Enhanced Chemical Stability of AdoMet Analogues for Improved Methyltransferase-Directed Labeling of DNA. ACS Chem. Biol. 2013, 8:1134-1139.

28. Huber TD, Wang F, Singh S, Johnson BR, Zhang J, Sunkara M, Van Lanen SG, Morris AJ, Phillips GN, Thorson JS: Functional AdoMet isosteres resistant to classical AdoMet degradation pathways. ACS ChemBio 2016, 11:acschembio.6b00348.

29. Najmanová L, Kutejová E, Kadlec J, Polan M, Olšovská J, Benada O, Novotná J, Kameník Z, Halada P, Bauer J, et al.: Characterization of $\mathbf{N}$-Demethyllincosamide Methyltransferases LmbJ and CcbJ. ChemBioChem 2013, 14:2259-2262.

30. Bauer J, Ondrovičová G, Najmanová L, Pevala V, Kameník Z, Koštan J, Janata J, Kutejová E: Structure and possible mechanism of the CcbJ methyltransferase from Streptomyces caelestis. Acta Crystallogr. Sect. D Biol. Crystallogr. 2014, 70:943-957.

31. Lee J, Hao Y, Blair PM, Melby JO, Agarwal V, Burkhart BJ, Nair SK, Mitchell DA: Structural and functional insight into an unexpectedly selective $\mathrm{N}$-methyltransferase involved in plantazolicin biosynthesis. Proc. Natl. Acad. Sci. U. S. A. 2013, 110:12954-9.

32. Hao Y, Blair PM, Sharma A, Mitchell DA, Nair SK: Insights into Methyltransferase Specificity and Bioactivity of Derivatives of the Antibiotic Plantazolicin. ACS Chem. Biol. 2015, 10:12091216.

33. Torres MA, Hoffarth E, Eugenio L, Savtchouk J, Chen X, Morris J, Facchini PJ, Ng KKS: Structural and Functional Studies of Pavine N-Methyltransferase from Thalictrum flavum Reveal Novel Insights into Substrate Recognition and Catalytic Mechanism.. J. Biol. Chem. 2016, doi:10.1074/jbc.M116.747261.

34. Robin $A Y$, Giustini $C$, Graindorge $M$, Matringe $M$, Dumas R: Crystal structure of norcoclaurine-6- $O$-methyltransferase, a key rate-limiting step in the synthesis of benzylisoquinoline alkaloids. Plant J. 2016, 87:641-653.

35. Sato F, Tsujita T, Katagiri Y, Yoshida S, Yamada Y: Purification and characterization of Sadenosyl-L-methionine:Norcoclaurine 6-0-methyltransferase from cultured coptis japonica cells. Eur. J. Biochem. 1994, 225:125-131.

36. Chatterjee D, Kudlinzki D, Linhard V, Saxena K, Schieborr U, Gande SL, Wurm JP, Hnert JW, Abele R, Rogov V V., et al.: Structure and biophysical characterization of the Sadenosylmethionine-dependent O-methyltransferase PaMTH1, a putative enzyme accumulating during senescence of Podospora anserina. J. Biol. Chem. 2015, 290:1641516430.

37. Averbeck NB, Jensen ON, Mann M, Schägger $H$, Osiewacz HD: Identification and characterization of PaMTH1, a putative O-methyltransferase accumulating during senescence of Podospora anserina cultures. Curr. Genet. 2000, 37:200-8.

38. Baptista FI, Henriques AG, Silva AMS, Wiltfang J, Da Cruz E Silva OAB: Flavonoids as therapeutic compounds targeting key proteins involved in Alzheimer's disease. ACS Chem. Neurosci. 2014, 5:83-92.

39. Creveling CR, Morris N, Shimizu H, Ong HH, Daly J: Catechol O-methyltransferase. IV. Factors affecting $\mathbf{m}$ - and $\mathrm{p}$-methylation of substituted catechols. Mol. Pharmacol. 1972, 8:398-409.

40. Li K, Frost JW: Synthesis of vanillin from glucose. J. Am. Chem. Soc. 1998, 120:10545-10546. 41. Hou X, Wang Y, Zhou Z, Bao S, Lin Y, Gong W: Crystal structure of SAM-dependent O- 
methyltransferase from pathogenic bacterium Leptospira interrogans. J. Struct. Biol. 2007, 159:523-528.

42. Ferrer J-LJ, Zubieta C, Dixon RRA, Noel JJP: Crystal structures of alfalfa caffeoyl coenzyme A 3-0-methyltransferase. Plant Physiol. 2005, 137:1009-1017.

43. Gregory M a, Hong H, Lill RE, Gaisser S, Petkovic H, Low L, Sheehan LS, Carletti I, Ready SJ, Ward MJ, et al.: Rapamycin biosynthesis: Elucidation of gene product function. Org. Biomol. Chem. 2006, 4:3565-3568.

44. Mahlert C, Kopp F, Thirlway J, Micklefield J, Marahiel MA: Stereospecific enzymatic transformation of $\alpha$-ketoglutarate to (2S,3R)-3-methyl glutamate during acidic lipopeptide biosynthesis. J. Am. Chem. Soc. 2007, 129:12011-12018.

45. Braun SD, Hofmann J, Wensing A, Ullrich MS, Weingart H, Völksch B, Spiteller D: Identification of the biosynthetic gene cluster for 3-methylarginine, a toxin produced by Pseudomonas syringae pv. syringae 22d/93 $\nabla$. Appl. Environ. Microbiol. 2010, 76:25002508.

46. Zou Y, Fang Q, Yin H, Liang Z, Kong D, Bai L, Deng Z, Lin S: Stereospecific biosynthesis of $\beta$ methyltryptophan from L-tryptophan features a stereochemical switch. Angew. Chemie Int. Ed. 2013, 52:12951-12955.

47. Zou X, Liu Y-C, Hsu N-S, Huang C, Lyu S-Y, Chan H, Chang C, Yeh H-W, Lin K, Wu C-J, et al.: Structure and mechanism of a nonhaem-iron SAM-dependent $C$-methyltransferase and its engineering to a hydratase and an 0 -methyltransferase. Acta Crystallogr. Sect. D Biol. Crystallogr. 2014, 70:1549-1560.

48. Koeller KM, Wong CH: Enzymes for chemical synthesis. Nature 2001, 409:232-240.

49. Crans DC, Whitesides GM: A convenient Synthesis of Disodium Acetyl Phosphate for Use in in Situ ATP Cofactor Regeneration. J. Org. Chem. 1983, 48:3130-3134.

50. Wong C, Drueckhammer DG, Sweers HM: Enzymatic vs. fermentative synthesis: thermostable glucose dehydrogenase catalyzed regeneration of $\mathrm{NAD}(\mathrm{P}) \mathrm{H}$ for use in enzymatic synthesis. J. Am. Chem. Soc. 1985, 107:4028-4031.

51. Wichmann R, Wandrey $C$, Bückmann AF, Kula MR: Continuous enzymatic transformation in an enzyme membrane reactor with simultaneous $N A D(H)$ regeneration. Biotechnol. Bioeng. 1981, 23:2789-2802.

52. Lee LG, Whitesides GM: Preparation of optically active 1,2-diols and $\boldsymbol{\alpha}$-hydroxy ketones using glycerol dehydrogenase as catalyst. Limits to enzyme-catalyzed synthesis due to noncompetitive and mixed inhibition by product. J. Org. Chem. 1986, 51:25-36.

53. Wang R, Luo M: A journey toward Bioorthogonal Profiling of Protein Methylation inside living cells. Curr. Opin. Chem. Biol. 2013, 17:729-737.

54. Zhang Y, Pan Y, Liu W, Zhou YJ, Wang K, Wang L, Sohail M, Ye M, Zou H, Zhao ZK: In vivo protein allylation to capture protein methylation candidates. Chem. Commun. 2016, 52:6689-6692.

55. Bennett B, Kimball E, Gao M: Absolute metabolite concentrations and implied enzyme active site occupancy in Escherichia coli. Nat. Chem. ... 2009, 5:593-599.

56. Chankhamjon $P$, Tsunematsu $Y$, Ishida-Ito $M$, Sasa $Y$, Meyer F, Boettger-Schmidt D, Urbansky B, Menzel K, Scherlach K, Watanabe K, et al.: Regioselective Dichlorination of a NonActivated Aliphatic Carbon Atom and Phenolic Bismethylation by a Multifunctional Fungal Flavoenzyme. Angew. Chemie 2016, 8526:1-6.

Special interest and outstanding interest papers

- Bauer, J. et al., 2014. 
The authors determined the crystal structure of the $\mathrm{CcbJ}$ MTase, highlighting the substrate binding pocket of the lincosamide antibiotic precursor $N$-demethylcelesticetin.

- Hao, Y. et al., 2015.

Using a structure guided approach, Hao et al use the BamL MTase to methylate a range of analogs of the antibiotic plantazolicin.

- Huber, T.D. et al., 2016.

Using the MAT enzyme, the authors produce a much more stable isostere of AdoMet; 7dzAdoMet resistant to two AdoMet degradation pathways.

•• Kim, J. 2013.

The authors are the first to discover a AdoMet analog that is naturally produced by an enzyme (CmoA). The discovery of $\mathrm{CmoA}$ producing carboxy-AdoMet offers an alternative approach to MAT produced AdoMet analogues, which could expand the range of AdoMet analogs currently available.

- Kunjapur, A.M., 2016.

This study is the first method to use deregulation of AdoMet biosynthesis to enhance vanillin producing in vivo, building on the early work of Li et al.

$\bullet \bullet$ Law, B.J.C. et al., 2016.

The authors improved the regioselectivity of COMT through structure-guided mutagenesis, as well investigating the regioselectivity of oligomeric forms of the COMT enzyme.

-Lee, J. et al., 2013

This work uncovered the crystal structure, for BamL, an $\mathrm{N}$-terminal dimethyl transferase that produces the antibiotic plantazolicin. The structure of BamL led to an understanding of the selectivity of the enzyme that enabled future researchers (Hao et al) to generate plantazolicin analogs.

•• Singh, S. et al., 2014

Singh et al's work utilised 5 MATs from a variety of organisms to generate a library of AdoMet analogs enzymatically. Despite earlier works demonstrating that MAT could be used to make 
AdoMet analogs, this study was first to demonstrate how a wide range of enzymatically produced AdoMet analogs could be used in MTase biocatalytic alkylation reactions.

•• Struck, A.W. et al., 2016

Using a mammalian catechol- $O$-methyltransferase and a fungal tyrosinase the authors were able to selectively methoxylate and alkoxylate a range of phenolic groups in peptides and proteins. This approach was used to alkoxylate the prostate cancer drug goserelin and the work could be further utilised in the field of site-selective protein labelling.

$\bullet$ Zou, X. et al., 2014.

Using crystallography, the authors solved the structure of the MppJ MTase enzyme providing the first example of non-haem iron-dependent AdoMet MTase and demonstrating potential biocatalytic applications of the MppJ enzyme. 
A

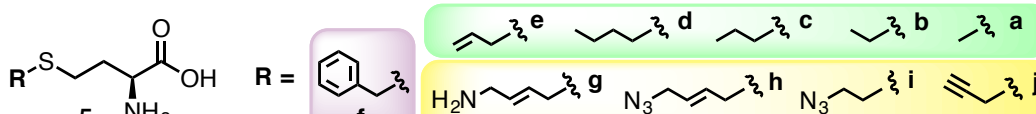

$$
\begin{aligned}
& 5 \mathrm{NH}_{2}
\end{aligned}
$$

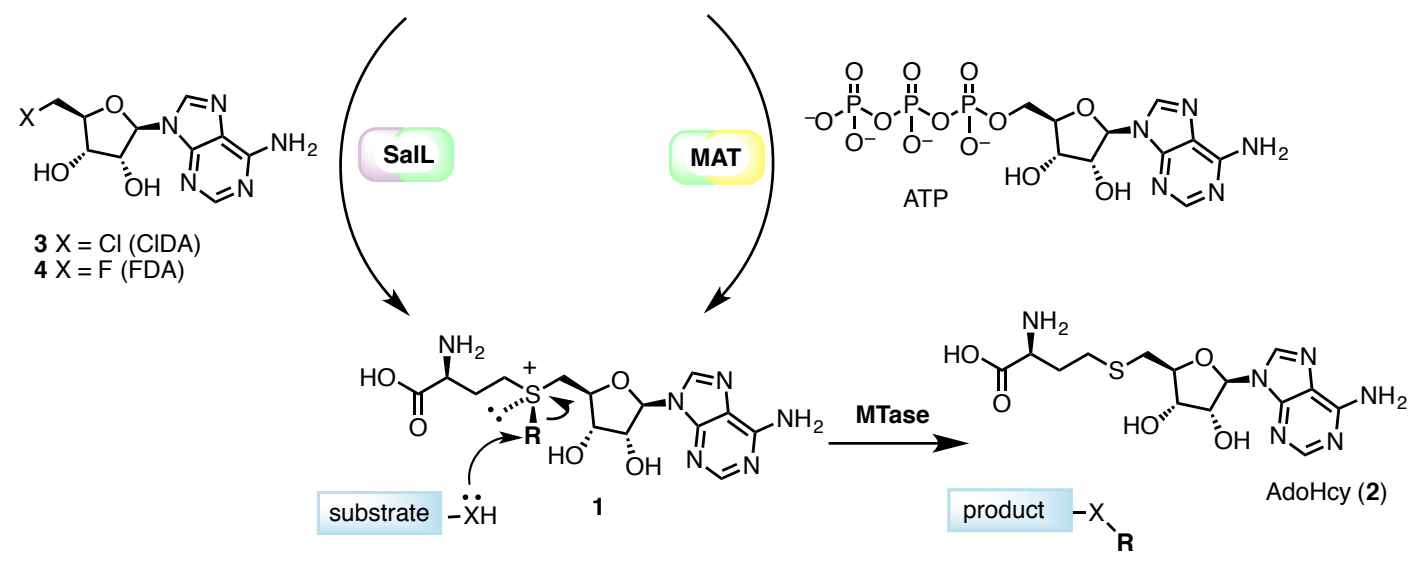

B<smiles>CCC(C)CC</smiles>

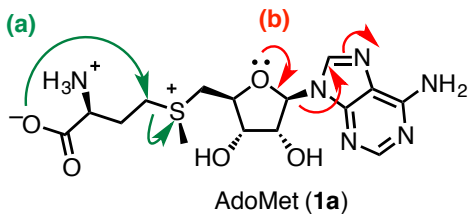

(b)<smiles>[3H]O[C@@H]1[C@H](C[Si](C)(C)CC[C@H]([NH3+])C(=O)OC)O[C@H](O)[C@@H]1O</smiles><smiles></smiles>

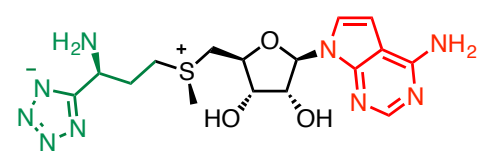

7dzAdotMet (10)

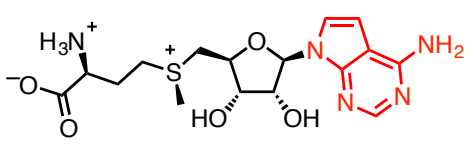

7dzAdoMet (9)

Figure 1: (A) Enzymatic AdoMet analog synthesis and subsequent MTase catalysed reactions. General scheme for the enzymatic synthesis of AdoMet (1a) and AdoMet analogs (1)-m), from L-Met (5a) and L-Met derivatives (5b-m) catalysed by either SalL (pink \& green substrates) or hMAT2A (yellow \& green substrates). (B) The two primary degradation pathways of AdoMet are side chain cyclisation (geen) or depurination (red) and to overcome co-factor degradation, more stable AdoMet isosteres AdotMet (8), 7dzAdoMet (9) and 7dzAdotMet (10) have been developed. 
A

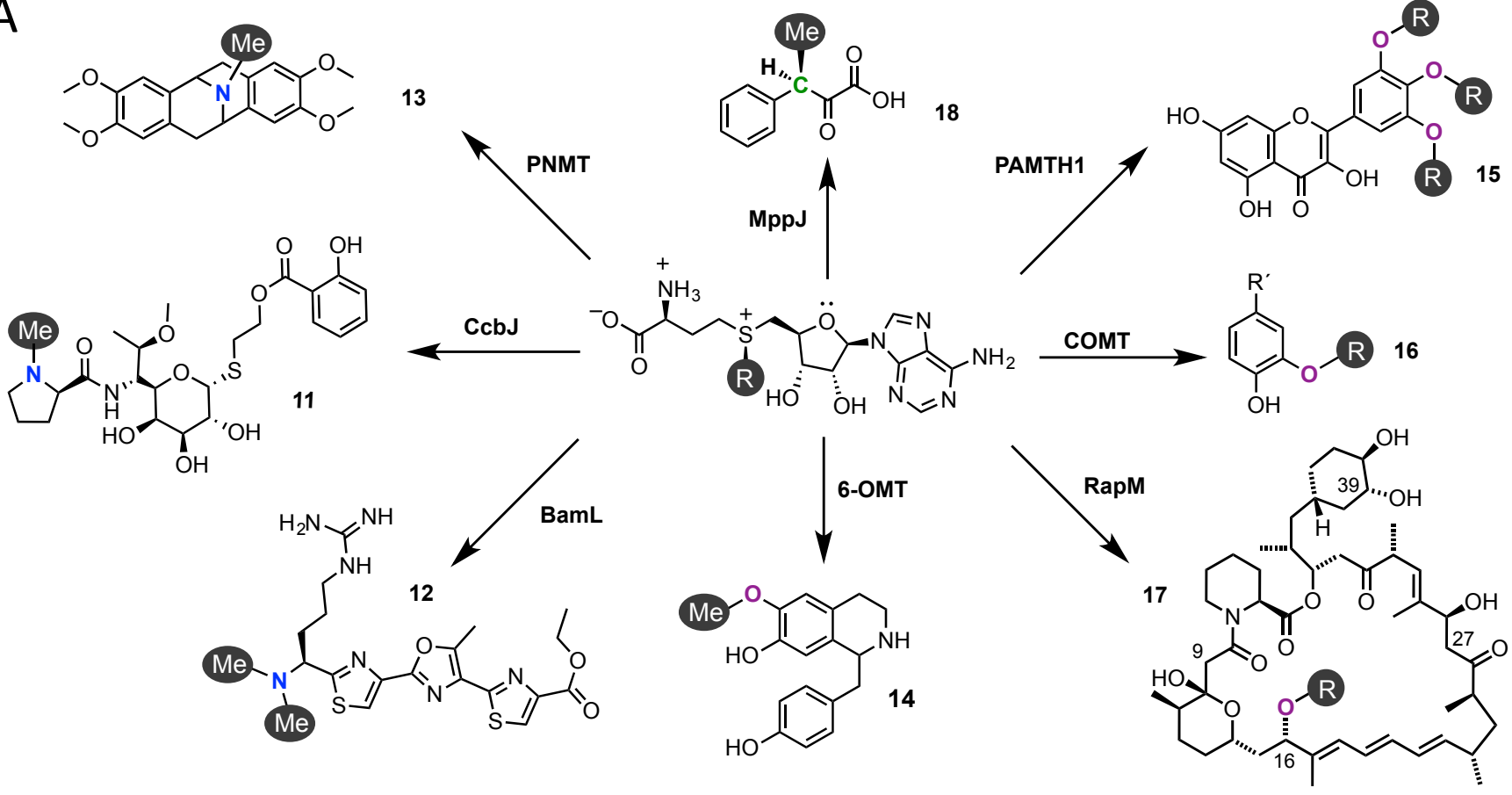

B

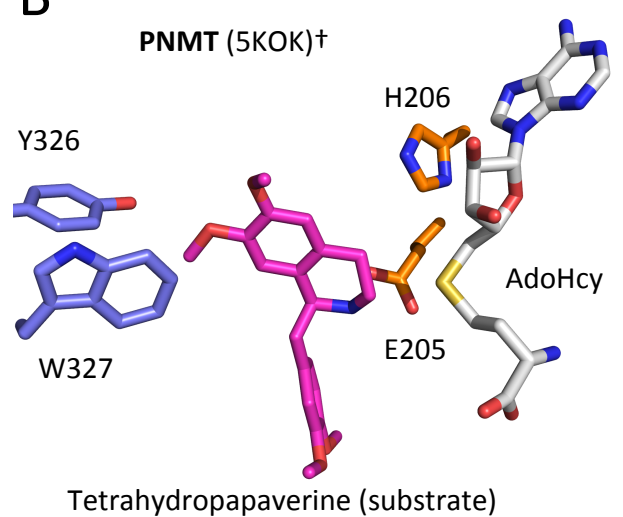

Tetrahydropapaverine (substrate)

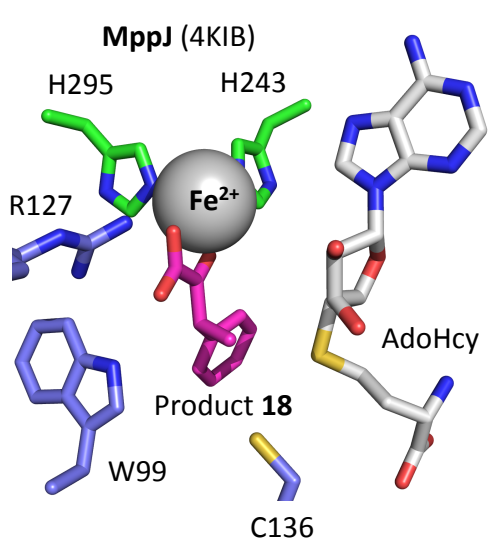

BamL (5DLY)

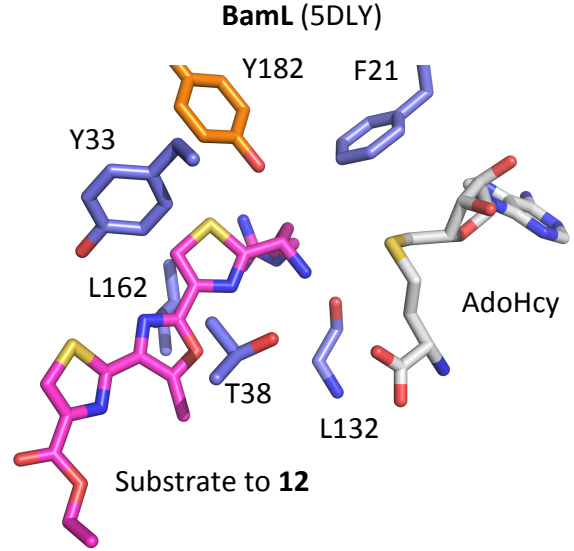

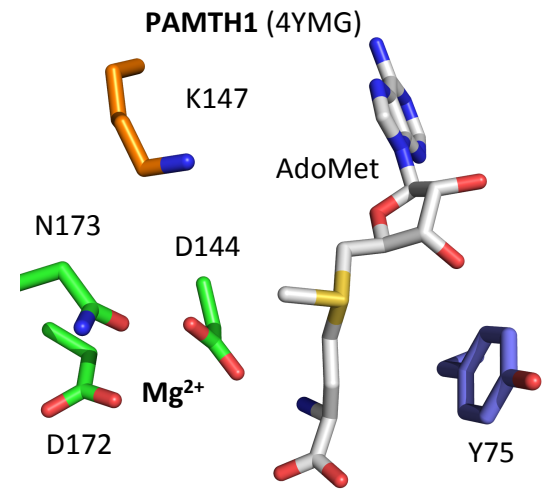

6-OMT (5ICE)

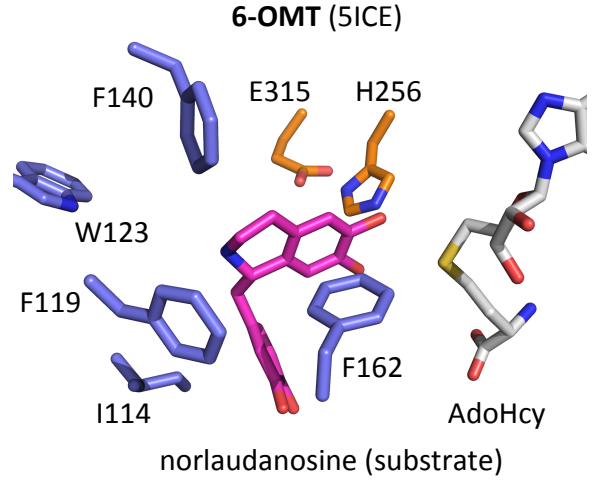

Figure 2: (A) Overview of AdoMet dependent MTase catalysed $\mathrm{O}-, \mathrm{N}$ - and $\mathrm{C}$ - methylation (Me) and alkylation (R) reactions. $R$ indicates the transfer of an alternative $S$-alkyl group from an AdoMet analogue, with the exception of the products of PAMTH1 where the position of mono-methylation has not yet been determined ( $R=H$ or $M e)$. (B) Active site architecture of MTase from recent X-ray crystalography studies showing: AdoMet or AdoHcy coenzymes (grey); Substrate or product (magenta), for PAMTH1 and CcbJ structures no ligand is bound; Key residues forming the substrate binding pocket (blue); residues pridicted to deprotonate the substrate (orange); residues coordinating metal ions. TPDB codes are in parentheses. 
A<smiles>COc1ccc(C=O)cc1O</smiles>

B

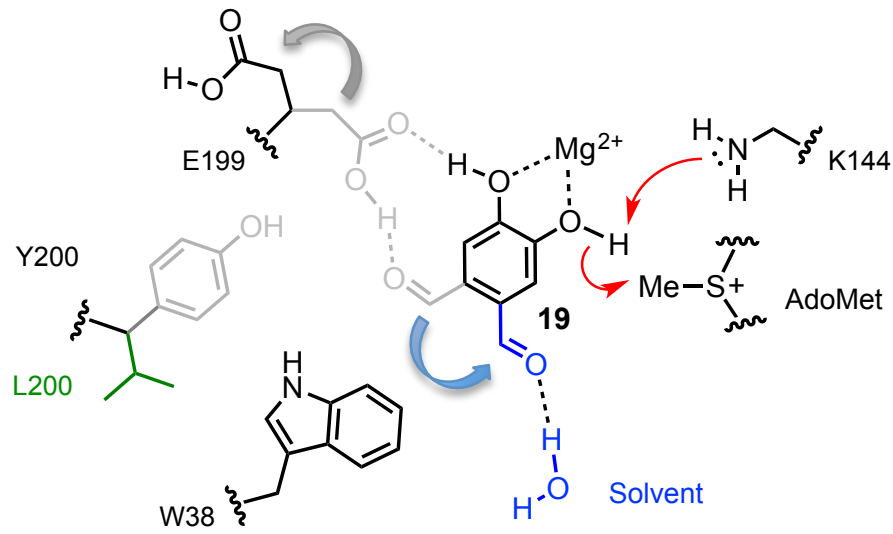

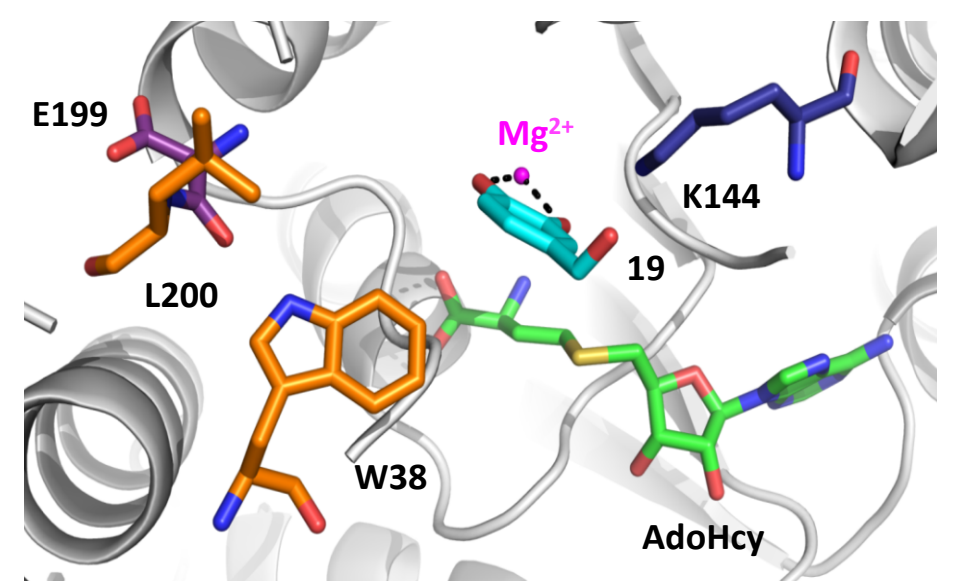

Figure 3: (A) COMT methylates the catechol substrate (19) leading to vanillin (20) and isovanillin (21) products. (B) COMT Y200L X-ray crystal structure showing the key residues W38 (orange), K144 (dark blue), E199 (purple, position the same in the WT COMT dimer not shown), Y200L (orange) and substrate 19 (cyan). Above the crystal structure is a cartoon showing the factors effecting the regioselectivity of COMT. For the Y200L mutant and WT COMT dimer E199 is orientated away from the active site which disfavours para-methylation resulting predominantely in the meta-methylated product vanillin (20). The Y200L mutation is indicated in green, whilst blue indicates prefered substrate binding orientation (for meta-methylation leading to 20) in the Y200L mutant and WT COMT dimer. The relative positions of the active site residues and substrate (19) in the WT COMT monomer structure are shown in light grey. In this case E199 is orientated into the active site leading to an alternative substrate binding mode that gives rise to higher levels of para-methylation leading to isovanillin (21). 
A<smiles>CC(C)Cc1cc(O)c(O)cc1CC(C)NC(C)C</smiles>

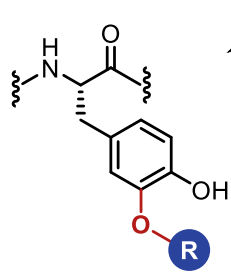

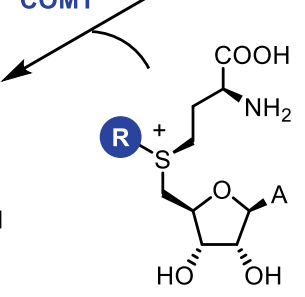<smiles>C#CCOC/C=C/COc1cc(C[C@H](NC(=O)[C@H](CO)NC(=O)[C@H](Cc2c[nH]c3ccccc23)NC(=O)[C@H](Cc2c[nH]cn2)NC(=O)C2CCC(=O)N2)NC(=O)[C@H](COC(C)(C)C)NC(=O)[C@H](CC(C)C)NC(=O)[C@H](CCCNC(=N)N)C(=O)N2CCC[C@H]2C(=O)NNC(N)=O)ccc1O</smiles>

B
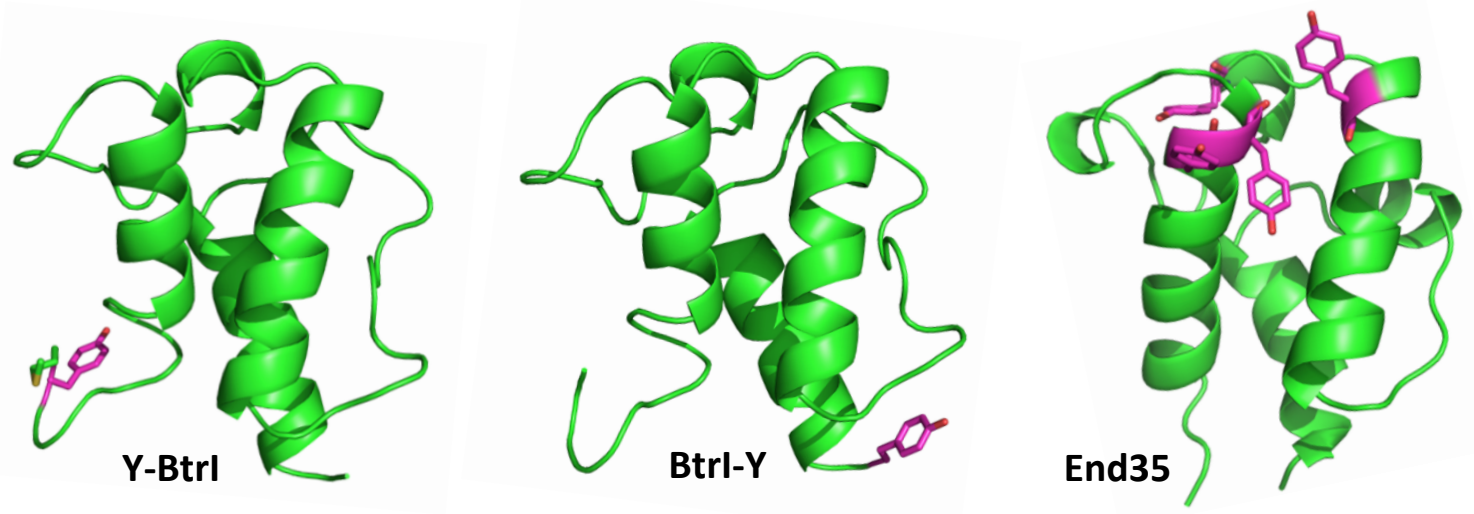

Figure 4: (A) General scheme for alkyl diversification of substrates possessing a phenolic moiety. (B) Selective alkoxylation of the anticancer drug goserelin, using tyrosinase, COMT and AdoMet analog $1 \mathrm{~m}$ (Figure $1 \mathrm{~A}$ ) to generate (22). (C) $N$ - and $C$ - terminal phenolic residues of Btrl were selectively methoxylated, whilst control protein End35 with internal phenolic residues was not methoxylated. 

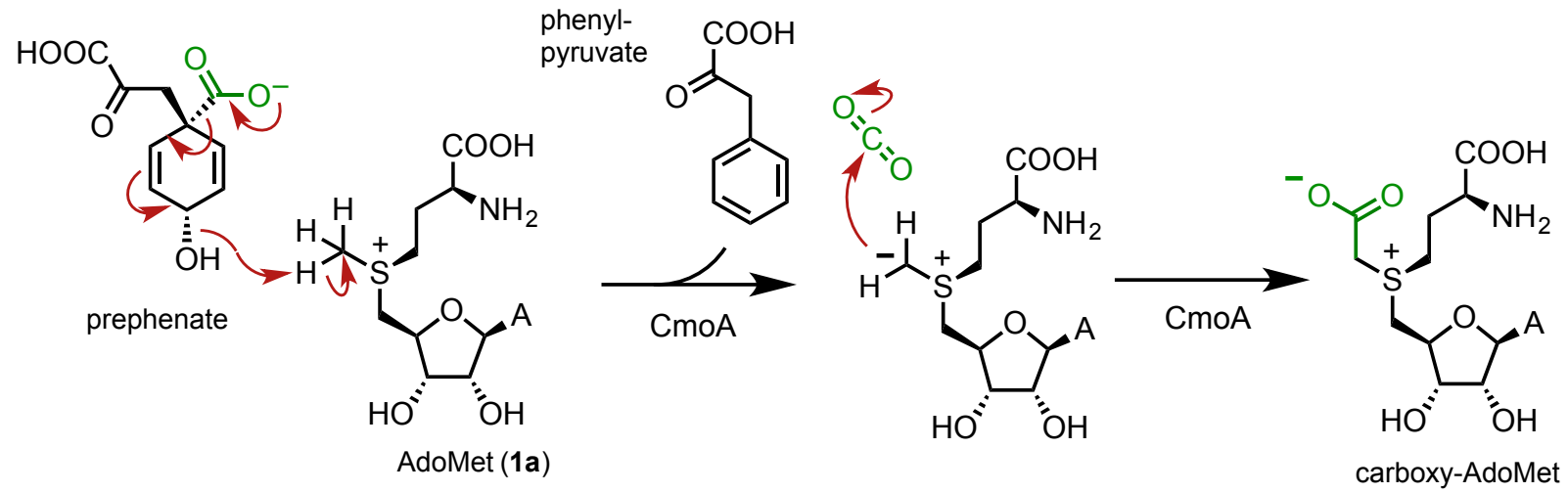

(23)

Figure 5. Proposed mechanism for the biosynthesis of carboxy-AdoMet (23) catalysed by the enzyme $\mathrm{CmoA}$. AdoMet protonates the prephenate hydroxyl group as it is eliminated during decarboxylation to give phenylpyruvate and an AdoMet sulfonium ylide. The nucleophilic ylide then attacks the carbon dioxide held in the active site to generate carboxy-AdoMet (23) . 